Document downloaded from:

http://hdl.handle.net/10251/60194

This paper must be cited as:

Lebtahi Ep-Kadi-Hahifi, L.; Patricio, P.; Thome, N. (2014). The diamond partial order in rings. Linear and Multilinear Algebra. 62(3):386-395. doi:10.1080/03081087.2013.779272.

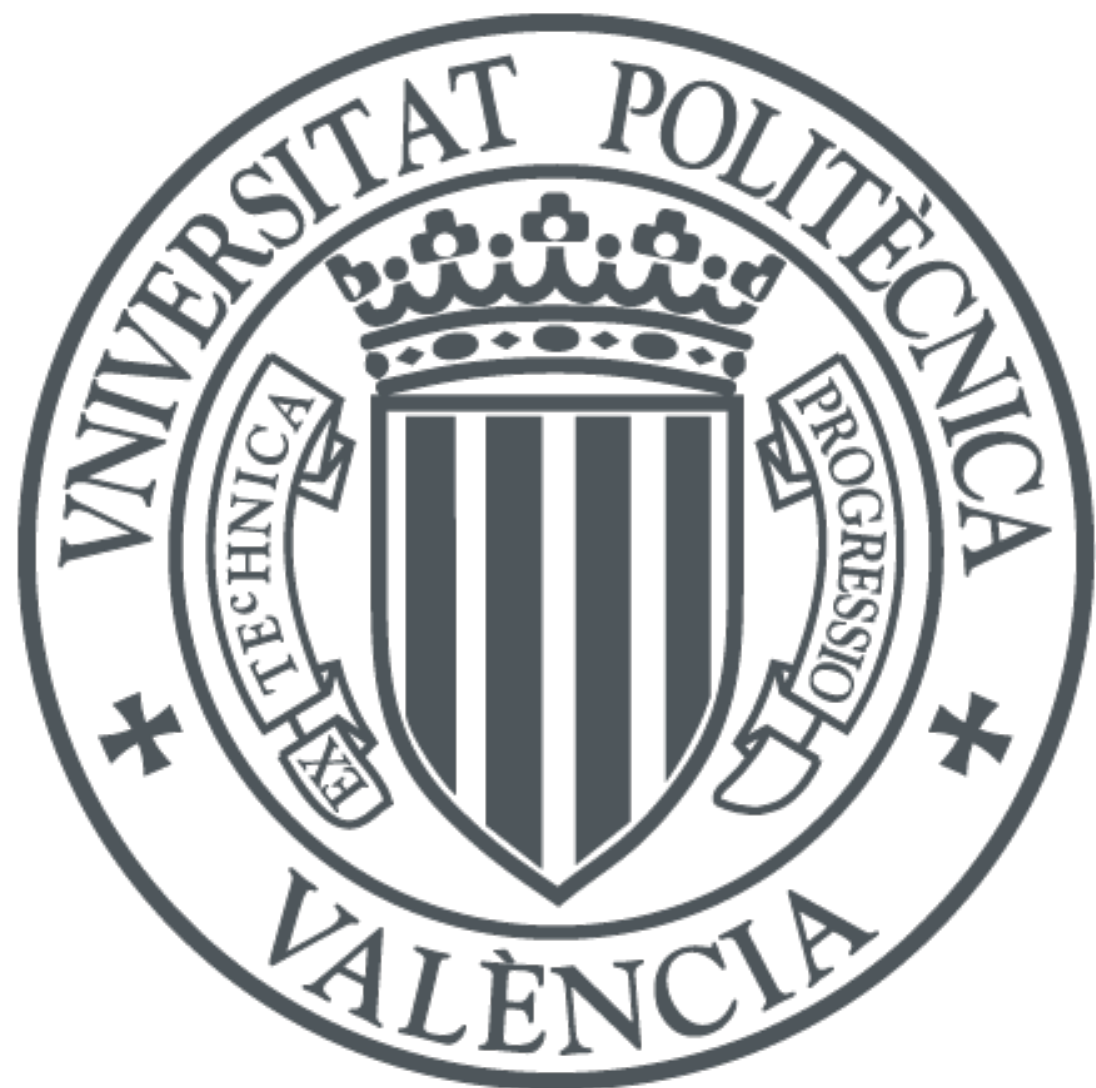

The final publication is available at

http://dx.doi.org/10.1080/03081087.2013.779272

Copyright Taylor \& Francis: STM, Behavioural Science and Public Health Titles

Additional Information

This is an author's accepted manuscript of an article published in " Linear and Multilinear Algebra"; Volume 62, Issue 3, 2014; copyright Taylor \& Francis; available online at: http://dx.doi.org/10.1080/03081087.2013.779272 


\title{
The diamond partial order in rings
}

\author{
Leila Lebtahi* $\quad$ Pedro Patrício ${ }^{\dagger} \quad$ Néstor Thome*
}

\begin{abstract}
In this paper we introduce a new partial order on a ring, namely the diamond partial order. This order is an extension of a partial order defined in a matrix setting in [J.K. Baksalary and J. Hauke, A further algebraic version of Cochran's theorem and matrix partial orderings, Linear Algebra and its Applications, 127, 157-169, 1990]. We characterize the diamond partial order on rings and study its relationships with other partial orders known in the literature. We also analyze successors, predecessors and maximal elements under the diamond order.
\end{abstract}

Keywords: star partial order, minus partial order, sharp partial order, ring, principal ideal.

AMS subject classification: Primary: 15A09; Secondary: 06A06

\section{Introduction and Background}

Let $R$ be an associative ring with unity 1 . For a given $a \in R$, we will denote

$$
a\{1\}:=\{x \in R: a x a=a\}
$$

*Instituto Universitario de Matemática Multidiscilpinar. Universitat Politècnica de València. E-46022 Valencia, Spain. E-mail: \{leilebep,njthome\}@mat.upv.es. These authors have been partially supported by grant DGI MTM2010-18228 and the third one by Universidad Nacional de La Pampa, Facultad de Ingeniería (grant Resol. No 049/11).

†Departamento de Matemática e Aplicações, CMAT-Centro de Matemática, Universidade do Minho, 4710-057, Braga, Portugal. E-mail: pedro@math.uminho.pt. This author was financed by FEDER Funds through "Programa Operacional Factores de Competitividade - COMPETE" and by Portuguese Funds through FCT - "Fundação para a Ciência e a Tecnologia", within the project PEst-C/MAT/UI0013/2011. 
the set of all $\{1\}$-inverses of $a$. A particular $\{1\}$-inverse of $a$ will be written as $a^{-}$, and the element $a$ is regular if $a\{1\} \neq \emptyset$. As usual, $R$ is a regular ring if all elements of $R$ are regular. A $\{1,2\}$-inverse of $a$ is a $\{1\}$-inverse of $a$ that is a solution of the ring equation $x a x=x$, it will be denoted by $x \in a\{1,2\}$. The unique $\{1,2\}$-inverse of $a$ that commutes with $a$ is called the group inverse of $a$ (when it exists) and denoted by $a^{\#}$. The set of group invertible elements is denoted by $R^{\#}$.

An involution $*$ in $R$ is an anti-isomorphism of degree 2 in $R$, that is to say, $\left(x^{*}\right)^{*}=x,(x+y)^{*}=x^{*}+y^{*}$ and $(x y)^{*}=y^{*} x^{*}$, for all $x, y \in R$. We will use the following notation: $a R=\{a x: x \in R\}$ and $R a=\{x a: x \in R\}$ the principal ideals; ${ }^{\circ}(a)=\{x \in R: x a=0\}$ and $(a)^{\circ}=\{x \in R: a x=0\}$.

We say $a \in R$ is Moore-Penrose invertible (with respect to $*$ ) if the equations $a x a=a, x a x=x,(a x)^{*}=a x,(x a)^{*}=x a$ have a common solution. If such a solution exists, then it is unique, and denoted by $a^{\dagger}$. The set of Moore-Penrose invertible elements is denoted by $R^{\dagger}$.

We recall some well-known partial orders on a regular ring $R$ :

- the minus partial order: $a \leq^{-} b$ iff $a^{-} a=a^{-} b$ and $a a^{-}=b a^{-}$.

- the star partial order: $a \leq^{*} b$ iff $a^{*} a=a^{*} b$ and $a a^{*}=b a^{*}$, which in turn is equivalent to $a^{\dagger} a=a^{\dagger} b$ and $a a^{\dagger}=b a^{\dagger}$ in $R^{\dagger}$.

- the left star partial order: $a * \leq b$ iff $a^{*} a=a^{*} b$ and $a R \subseteq b R$.

- the right star partial order: $a \leq * b$ iff $a a^{*}=b a^{*}$ and $R a \subseteq R b$.

- the sharp partial order in $R^{\#}: a \leq^{\#} b$ iff $a^{\#} a=a^{\#} b$ and $a a^{\#}=b a^{\#}$.

- the direct sum partial order: $a \leq^{\oplus} b$ iff $b R=a R \oplus(b-a) R$.

A detailed analysis of these partial orders has been done in [8] for a matrix approach.

Throughout this paper, $R$ will be a ring with involution and we will assume $R$ is ${ }^{*}$-regular, i.e., all elements have a Moore-Penrose inverse.

We define (see [3])

$$
a \leq_{\diamond} b \quad \text { iff } \quad a R \subseteq b R, R a \subseteq R b \text { and } a a^{*} a=a b^{*} a .
$$

In Section 3 we are going to prove that the binary relation $\leq_{\diamond}$ defines a partial order on $R$ and, from now on, it is called the diamond partial order. It should 
be mentioned that the diamond partial order has not been considered in the literature, as far as we know, in the setting of rings.

We recall some well-known facts.

Lemma 1 Let $a \in R$ and $a^{-}, a^{=} \in a\{1\}$. Then $a^{-} a a^{=} \in a\{1,2\}$.

Lemma 2 Let $a, b \in R$. Then

(a) $a \leq^{-} b$ iff there exists $b^{-}$such that $b b^{-} a=a b^{-} b=a b^{-} a=a$.

(b) $a R \subseteq b R$ iff $R a^{*} \subseteq R b^{*}$ and $R a \subseteq R b$ iff $a^{*} R \subseteq b^{*} R$.

(c) $a^{*} R=a^{\dagger} R$ and $R a^{*}=R a^{\dagger}$.

Proof. (a) $(\Longrightarrow)$ By hypothesis we have: $a^{-} a=a^{-} b$ and $a a^{-}=b a^{-}$. Then

$$
\begin{aligned}
& b b^{-} a=b b^{-} a a^{-} a=b b^{-} b a^{-} a=b a^{-} a=a a^{-} a=a, \\
& a b^{-} b=a a^{-} a b^{-} b=a a^{-} b b^{-} b=a a^{-} b=a a^{-} a=a,
\end{aligned}
$$

and

$$
a b^{-} a=a a^{-} a b^{-} a a^{-} a=a a^{-} b b^{-} b a^{-} a=a a^{-} b a^{-} a=a a^{-} a a^{-} a=a .
$$

$(\Longleftarrow)$ We note that $b^{-}$is a $\{1\}$-inverse of $a$ since $a b^{-} a=a$. The equalities $a=b a^{-} a=a a^{-} b$ hold by taking $a^{-}=b^{-}$since $a=b b^{-} a=a b^{-} b$.

(b) Trivial by definition.

(c) It follows from the properties $a^{*}=a^{\dagger}\left(a a^{*}\right)=\left(a^{*} a\right) a^{\dagger}$ and $a^{\dagger}=$ $a^{*}\left(a a^{*}\right)^{\dagger}=\left(a^{*} a\right)^{\dagger} a^{*}$.

We remark that

(I) $a b^{\dagger} a=a \Longrightarrow a b^{\dagger}$ and $b^{\dagger} a$ are idempotent.

(II) $b b^{\dagger} a=a \Longleftrightarrow b b^{\dagger} a a^{\dagger}=a a^{\dagger}$.

(III) $a b^{\dagger} b=a \Longleftrightarrow a^{\dagger} a b^{\dagger} b=a^{\dagger} a$.

(IV) If the equalities $b b^{-} a=a b^{-} b=a b^{-} a=a$ hold for some $b^{-}$then they hold for any choice of $b^{-}$. Indeed, the independence of $b^{-}$in $b b^{-} a=a b^{-} b=a$ follows directly from [9, Lemma 2.1]. For a $\{1\}-$ inverse $b^{-}$of $b$, it is well known [10, pp. 26] that all $\{1\}$-inverses of $b$ are of the form $b^{=}=b^{-}+\left(1-b^{-} b\right) h+z\left(1-b b^{-}\right)$for some choice of $h$ and $z$. As $b b^{-} a=a b^{-} b=a b^{-} a=a$ it follows that $a b^{=} a=a$. To sum up we showed the independence of the equalities $b b^{-} a=a b^{-} b=a b^{-} a=a$ to the choice of $b^{-}$. 
Lemma 3 [4, Corollary 4] Let $x, y \in R$ such that $y$ is idempotent. Then $x \leq^{-} y$ iff $x=x^{2}=x y=y x$.

Proof. For the sake of completeness we include a proof.

$(\Longrightarrow)$ Since $x x^{-}=y x^{-}$and $x^{-} x=x^{-} y$, we get $x=x x^{-} y=y x^{-} x$. Then $x y=x x^{-} y^{2}=x x^{-} y=x$ and $y x=y^{2} x^{-} x=y x^{-} x=x$. Moreover, $x^{2}=x x^{-} y x=x x^{-} x y=x y$.

$(\Longleftarrow)$ From $x^{2}=x$ we have that $x$ is group invertible and $x^{\#}=x$. Taking $x^{-}=x^{\#}$ we get $x x^{-}=x^{2}=y x=y x^{-}$and $x^{-} x=x^{-} y$ is similar.

A wide range of properties related to these orders and the generalized inverses involved in each of them can be found in $[1,2,3,6,7,11,12,13]$.

This paper is organized as follows. In Section 2 we analyze some relationships between the diamond binary relation and the minus, left star, right star, star and sharp partial orders. In Section 3 the diamond partial order on rings is characterized. Section 4 is devoted to the study of successors and predecessors under the diamond order. In addition, maximal elements under the diamond partial order are found.

\section{Relations between the diamond order and other partial orders}

Firstly, we notice that the equivalence $a \leq_{\diamond} b \Longleftrightarrow b-a \leq_{\diamond} b$ does not hold for the diamond partial order (see an example in [3]) despite of it remains valid for the star and minus orders, as stated in the following result.

Lemma 4 Given regular $x, y \in R$,

(a) $x \leq^{-} y$ iff $y-x \leq^{-} y$.

(b) $x \leq^{*} y$ iff $y-x \leq^{*} y$.

Proof.(a) If $x \leq^{-} y$ then by [5, Proposition 3 (i)]

$$
y=x+\left(1-x x^{+}\right) s\left(1-x^{+} x\right)
$$

for some $\{1,2\}$-inverse $x^{+}$of $x$ and an arbitrary $s \in R$. Setting the idempotents $e=1-x x^{+}$and $f=1-x^{+} x$ there exists $(y-x)^{+}=f w e$ for some $w \in R$. For this choice, $(y-x)(y-x)^{+}=$esfwe $=(x+e s f) f w e=y(y-x)^{+}$. Similarly, $(y-x)^{+} y=f w e(x+e s f)=$ fwesf $=(y-x)^{+}(y-x)$. 
Conversely, if $y-x \leq^{-} y$ then by the previous implication $x=y-(y-$ $x) \leq^{-} y$.

(b) From $x \leq^{*} y$ we obtain the equalities $(y-x) x^{*}=x^{*}(y-x)=0$, from which $(y-x)(y-x)^{*}=(y-x) y^{*}$ and $(y-x)^{*}(y-x)=y^{*}(y-x)$. Since these are hermitian, the equalities $(y-x)(y-x)^{*}=y(y-x)^{*}$ and $(y-x)^{*}(y-x)=(y-x)^{*} y$ hold.

The converse follows from the above implication as in (a).

We also observe that in the diamond partial order the symbol $\dagger$ does not play the same role that ${ }^{*}$ as in the star partial order. That is, neither of the implications $a a^{*} a=a b^{*} a \Longrightarrow a a^{\dagger} a=a b^{\dagger} a$ (in $\mathbb{C}^{2 \times 2}$ take $a=$ $\left[\begin{array}{ll}1 & 1 \\ 0 & 0\end{array}\right]$ and $b=\left[\begin{array}{ll}2 & 0 \\ 0 & 2\end{array}\right]$ ) nor $a a^{\dagger} a=a b^{\dagger} a \Longrightarrow a a^{*} a=a b^{*} a$ (in $\mathbb{C}^{2 \times 2}$ take $a=\left[\begin{array}{ll}1 & 1 \\ 0 & 0\end{array}\right]$ and $\left.b=\left[\begin{array}{ll}1 & 0 \\ 0 & 1\end{array}\right]\right)$ is valid in general.

We remark that $*$ is isotone with respect to the diamond partial order. That is to say $a \leq_{\diamond} b$ exactly when $a^{*} \leq_{\diamond} b^{*}$. This follows from Lemma 2 . As a consequence we have the following proposition.

Proposition $1 a \leq_{\diamond}\left(a^{\dagger}\right)^{*}$ iff $a$ is a partial isometry (i.e., $\left.a^{\dagger}=a^{*}\right)$.

Proof. If $a \leq_{\diamond}\left(a^{\dagger}\right)^{*}$ then $a a^{*} a=a a^{\dagger} a=a$, that is $a^{*} \in a\{1\}$. Since $a a^{*}$ and $a^{*} a$ are hermitian and $a^{*} a a^{*}=a^{*}$, we get $a^{*}=a^{\dagger}$. The converse is trivial.

Some equivalent conditions to $a a^{*} a=a b^{*} a$ are given in the following result.

Lemma 5 Let $a, b \in R$. Then the following conditions are equivalent:

(a) $a a^{*} a=a b^{*} a$.

(b) $a^{\dagger} b a^{\dagger} \in a\{1\}$.

(c) $a^{\dagger} b a^{\dagger} \in a\{1,2\}$.

(d) $a^{\dagger} b a^{\dagger}=a^{\dagger}$.

\section{Proof.}

(a) $\Longrightarrow$ (b) Multiplying $a a^{*} a=a b^{*} a$ on the left and right sides by $a^{\dagger}$ we get $a^{*}=a^{\dagger} a a^{*} a a^{\dagger}=a^{\dagger} a b^{*} a a^{\dagger}=a^{*}\left(a^{\dagger} b a^{\dagger}\right)^{*} a^{*}$. Hence, $a^{\dagger} b a^{\dagger} \in a\{1\}$. 
(b) $\Longrightarrow$ (a) Multiplying $a\left(a^{\dagger} b a^{\dagger}\right) a=a$ on the left and right sides by $a^{*}$ item (a) can be easily obtained.

(b) $\Longrightarrow$ (c) It follows applying Lemma 1 with $a^{-}=a^{\dagger} b a^{\dagger}$ and $a^{=}=a^{\dagger}$.

(c) $\Longrightarrow(\mathrm{b})$ is trivial.

(a) $\Longrightarrow(\mathrm{d})$ Multiplying firstly both sides of $a^{*} a a^{*}=a^{*} b a^{*}$ by $\left(a^{\dagger}\right)^{*}$ and then both sides of the obtained result by $a^{\dagger}$ we get $a^{\dagger}=a^{\dagger} b a^{\dagger}$.

(d) $\Longrightarrow(\mathrm{c}) \Longrightarrow($ a) are trivial.

Theorem 1 Let $a, b \in R$. Then the following conditions are equivalent:

(a) $a \leq_{\diamond} b$

(b) $a R \subseteq b R, R a \subseteq R b$ and $a^{\dagger} b a^{\dagger} \in a\{1\}$.

(c) $a R \subseteq b R, R a \subseteq R b$ and $a^{\dagger} b a^{\dagger} \in a\{1,2\}$.

(d) $a R \subseteq b R, R a \subseteq R b$ and $a^{\dagger} b a^{\dagger}=a^{\dagger}$.

Proof. It follows by definition of the diamond partial order and Lemma 5 .

The implications $a * \leq b \Rightarrow a \leq_{\diamond} b$ and $a \leq_{\diamond} b \Rightarrow a * \leq b$ are not valid in general. Similarly, for $\leq *$ instead of $* \leq$.

Even for matrices over a field, the implication $a \leq * b \Rightarrow a \leq_{\diamond} b$ might not hold. Take, over the field $\mathbb{Z}_{13}$, the matrices $A=\left[\begin{array}{ll}9 & 7 \\ 2 & 3\end{array}\right]$ and $B=\left[\begin{array}{rr}0 & 0 \\ 10 & 2\end{array}\right]$, and the transposition as the involution. Then $\left[\begin{array}{ll}9 & 7\end{array}\right]=10\left[\begin{array}{ll}10 & 2\end{array}\right]$, $\left[\begin{array}{ll}2 & 3\end{array}\right]=8\left[\begin{array}{ll}10 & 2\end{array}\right]$ and row space of $A$ is a subspace of the row space of $B$. As $A A^{*}=B A^{*}$ then $A \leq * B$. Nevertheless, $\left[\begin{array}{l}9 \\ 2\end{array}\right]=\alpha\left[\begin{array}{c}0 \\ 10\end{array}\right]+\beta\left[\begin{array}{l}0 \\ 2\end{array}\right]$ has no solutions in $\mathbb{Z}_{13}$, and hence the column space of $A$ is not a subspace of the column space of $B$.

Needless to say a similar conclusion can be drawn for $* \leq$, as $A^{*} * \leq B^{*}$ and yet $A^{*} \underline{\Delta}_{\diamond} B^{*}$ since the row space of $A^{*}$ is not a subspace of the row space of $B^{*}$, where $A$ and $B$ are as the previous example.

Lemma 6 Let us consider the following statements:

(a) $a \leq_{\diamond} b$ 
(b) $a * \leq b$ and $R a \subseteq R b$.

(c) $a \leq * b$ and $a R \subseteq b R$.

Then $(b) \Longrightarrow(a)$ and $(c) \Longrightarrow(a)$.

Remark 1 Observe that in Lemma 6 neither $(a) \Longrightarrow(b)$ nor $(a) \Longrightarrow(c)$ as the following example allows us to check in $\mathbb{C}^{2 \times 2}$ :

$$
a=\left[\begin{array}{ll}
1 & 0 \\
0 & 0
\end{array}\right] \quad \text { and } \quad b=\left[\begin{array}{ll}
1 & 1 \\
1 & 0
\end{array}\right]
$$

Proposition 2 Let $a, b \in R$. Then

(a) $a \leq^{*} b \Longrightarrow a \leq_{\diamond} b$.

(b) $a \leq^{\#} b \Longrightarrow a^{\dagger} \leq_{\diamond} b^{\dagger} \Longleftrightarrow a \leq^{-} b$.

Proof. (a) Suppose $a \leq^{*} b$, that is, $a a^{*}=b a^{*}$ and $a^{*} a=a^{*} b$. Then $a^{*}(b-a) a^{*}=0$, from which $a a^{*} a=a b^{*} a$. Furthermore, post-multiplying $a a^{*}=b a^{*}$ and pre-multiplying $a^{*} a=a^{*} b$ by $\left(a^{\dagger}\right)^{*}$, we obtain $a=b a^{*}\left(a^{\dagger}\right)^{*}=$ $\left(a^{\dagger}\right)^{*} a^{*} b \in b R \cap R b$ and as such $a R \subseteq b R$ and $R a \subseteq R b$.

(b) It is well known that $a \leq^{\#} b \Longrightarrow a \leq^{-} b$ and this last expression is equivalent to $a^{\dagger} \leq_{\diamond} b^{\dagger}$ (as we will see in Theorem 2).

Note that, despite Theorem 2 has been not proved yet, we have included Proposition 2 in this section to collect all the relationships between the diamond partial order and the other ones.

Now, we remark that $a \leq_{\diamond} b$ does not imply $a \leq^{*} b$. A counterexample can be found by taking the real matrices

$$
a=\left[\begin{array}{ll}
1 & 0 \\
0 & 0
\end{array}\right], \quad b=\left[\begin{array}{ll}
1 & 1 \\
0 & 1
\end{array}\right] .
$$

We can also observe that $a \leq^{\#} b$ does not imply $a \leq_{\diamond} b$ as the following real matrices show:

$$
a=\left[\begin{array}{ll}
1 & 1 \\
0 & 0
\end{array}\right], \quad b=\left[\begin{array}{ll}
1 & 0 \\
0 & 1
\end{array}\right] .
$$

We close this section with the following remark. 
Remark 2 The condition on the Moore-Penrose invertibility of $a$ in (a) of the previous Proposition cannot be dropped. We will present an example using matrices over $\mathbb{Z}_{4}$ with the involution $*$ as transposition. Take $A=$ $\left[\begin{array}{ll}1 & 0 \\ 2 & 2\end{array}\right]$ and $B=\left[\begin{array}{ll}1 & 0 \\ 2 & 0\end{array}\right]$ which satisfy $A \leq^{*} B$, since $A A^{*}=B A^{*}, A^{*} A=$ $A^{*} B$. Yet, $A=B\left[\begin{array}{cc}x & z \\ y & w\end{array}\right]$ would imply $z=0$ and $0=2$, and therefore $A \leq_{\diamond} B$ does not hold. Note that $A^{\dagger}$ does not exist as the (free) $\mathbb{Z}_{4}$-module generated by the columns of $A$ is not a submodule of the generated by the columns of $A A^{*}$. Indeed, there are no solutions in $\mathbb{Z}_{4}$ for $\left[\begin{array}{l}0 \\ 2\end{array}\right]=\alpha\left[\begin{array}{l}1 \\ 2\end{array}\right]+$ $\beta\left[\begin{array}{l}2 \\ 0\end{array}\right]$.

\section{Characterizations of the diamond partial order}

Now, we characterize the diamond partial order in terms of the minus partial order.

Theorem 2 Let $a, b \in R$. Then the following statements are equivalent:

(a) $a \leq_{\diamond} b$

(b) $a^{\dagger} \leq^{-} b^{\dagger}$.

(c) $a a^{\dagger} b b^{\dagger}=a a^{\dagger}, b^{\dagger} b a^{\dagger} a=a^{\dagger} a, a^{\dagger} b a^{\dagger}=a^{\dagger}$.

Proof. (a) $\Longrightarrow$ (b) and (c) By hypothesis and Lemma 2 we have: $R a^{\dagger} \subseteq R b^{\dagger}$ and $a^{\dagger} R \subseteq b^{\dagger} R$. Since $a^{\dagger}=a^{\dagger} a a^{\dagger} \in a^{\dagger} R \cap R a^{\dagger} \subseteq b^{\dagger} R \cap R b^{\dagger}$.

Since $a^{\dagger}=b^{\dagger} x$ for some $x \in R, b^{\dagger} b a^{\dagger}=b^{\dagger} b b^{\dagger} x=b^{\dagger} x=a^{\dagger}$. Hence, $b^{\dagger} b a^{\dagger}=a^{\dagger}$ and so $b^{\dagger} b a^{\dagger} a=a^{\dagger} a$.

Since $a^{\dagger}=y b^{\dagger}$ for some $y \in R, a^{\dagger} b b^{\dagger}=y b^{\dagger} b b^{\dagger}=y b^{\dagger}=a^{\dagger}$. Hence, $a^{\dagger} b b^{\dagger}=a^{\dagger}$ and so $a a^{\dagger} b b^{\dagger}=a a^{\dagger}$.

Lemma 5 assures that the condition $a^{*} a a^{*}=a^{*} b a^{*}$ is equivalent to $a^{\dagger}=$ $a^{\dagger} b a^{\dagger}$. Finally, Lemma 2 implies that $a^{\dagger} \leq^{-} b^{\dagger}$ holds.

(b) $\Longrightarrow$ (a) From $a^{\dagger} a=b^{\dagger} a$ and $a a^{\dagger}=a b^{\dagger}$ we get $a^{\dagger}=b^{\dagger} a a^{\dagger} \in b^{\dagger} R$ and $a^{\dagger}=a^{\dagger} a b^{\dagger} \in R b^{\dagger}$. Then $a^{\dagger} R \subseteq b^{\dagger} R$ and $R a^{\dagger} \subseteq R b^{\dagger}$. Moreover, Lemma 2 (a) assures that $a^{\dagger}=a^{\dagger} b a^{\dagger}$. Finally, Lemma 5 implies that $a^{*} a a^{*}=a^{*} b a^{*}$. 
(c) $\Longrightarrow$ (a) From $a a^{\dagger} b b^{\dagger}=a a^{\dagger}$ we get $a^{\dagger} b b^{\dagger}=a^{\dagger}$, that is $a^{\dagger} \in R b^{\dagger}$. Thus, $R a^{*}=R a^{\dagger} \subseteq R b^{\dagger}=R b^{*}$ and this implies $a R \subseteq b R$.

Similarly, $b^{\dagger} b a^{\dagger} a=a^{\dagger} a$ yields $R a \subseteq R b$. Moreover, the equivalence between $a^{\dagger} b a^{\dagger}=a^{\dagger}$ and $a a^{*} a=a b^{*} a$ has been shown in Lemma 5. Hence, $a \leq_{\diamond} b$.

We remark that in Theorem 2 (c) the hypothesis $a^{\dagger} b a^{\dagger}=a^{\dagger}$ cannot be dropped. Take the real matrices, with transposition as involution, $A=$ $\left[\begin{array}{ll}1 & 1 \\ 0 & 0\end{array}\right]$ and $B=I_{2}$, for which $A \not \mathbb{\diamond}_{\diamond} B$ since $A A^{T} A \neq A^{2}=A$, and yet $A A^{\dagger} B B^{\dagger}=A A^{\dagger}, B^{\dagger} B A^{\dagger} A=A^{\dagger} A$, but $A^{\dagger} B A^{\dagger} \neq A^{\dagger}$.

Theorem 2 allows us to assure that the diamond relation is a partial order.

Corollary 1 The binary relation $\leq_{\diamond}$ is a partial order on the ring $R$.

Notice that neither of the implications $a \leq_{\diamond} b \Longrightarrow a \leq^{-} b$ nor $a \leq^{-} b \Longrightarrow$ $a \leq_{\diamond} b$ are valid in general (see examples in [3, pp. 165]).

We recall that $a \leq^{-} b$ iff there exist idempotents $e, f \in R$ such that $a=e b=b f$. This will lead to the following result.

Theorem 3 Let $a, b \in R$. Then the following conditions are equivalent:

(a) $a \leq_{\diamond} b$

(b) $\left(b^{\dagger}-a^{\dagger}\right)^{\dagger} \leq_{\diamond} b$.

(c) There exist idempotents $e, f \in R$ such that $a=\left(e b^{\dagger}\right)^{\dagger}=\left(b^{\dagger} f\right)^{\dagger}$.

Proof. The equivalences follow from the properties of the minus partial order, Theorem 2 and Lemma 4.

We remark that $a \leq_{\diamond} b$ does not imply $a^{\dagger} \leq_{\diamond} b^{\dagger}$, even though $a R \subseteq$ $b R$ and $R a \subseteq R b$ imply $R a^{\dagger}=R a^{*} \subseteq R b^{*}=R b^{\dagger}$ and $a^{\dagger} R=a^{*} R \subseteq$ $b^{*} R=b^{\dagger} R$. The implication is not valid as $a a^{*} a=a b^{*} a$ is not sufficient to $a^{\dagger}\left(a^{\dagger}\right)^{*} a^{\dagger}=a^{\dagger}\left(b^{\dagger}\right)^{*} a^{\dagger}$. Take the rational matrices, with the transposition as involution, $A=\left[\begin{array}{ll}1 & 0 \\ 0 & 0\end{array}\right]$ with $A^{\dagger}=\left[\begin{array}{ll}1 & 0 \\ 0 & 0\end{array}\right]$, and $B=\left[\begin{array}{ll}1 & 2 \\ 1 & 1\end{array}\right]$ with $B^{\dagger}=B^{-1}=$ $\left[\begin{array}{rr}-1 & 2 \\ 1 & -1\end{array}\right]$. Then $A \leq_{\diamond} B$ and yet $A^{\dagger} \leq_{\diamond} B^{-1}$. 
Theorem 4 Let $a, b \in R$. Then $a \leq_{\diamond} b \Longleftrightarrow a^{\dagger} \leq^{\oplus} b^{\dagger}$.

Proof. It is well known that $x \leq^{-} y$ iff $x \leq^{\oplus} y$ [4, Lemma 3]. Then, this item follows directly applying Theorem 2 with $x=a^{\dagger}, y=b^{\dagger}$.

Neither the implication $a \leq_{\diamond} b \Longrightarrow a \leq^{\oplus} b$ nor $a \leq^{\oplus} b \Longrightarrow a \leq_{\diamond} b$ are valid in general. Indeed, it follows from the fact that $x \leq^{-} y$ iff $x \leq^{\oplus} y$.

Moreover, for the matrices

$$
a=\frac{1}{2}\left[\begin{array}{ll}
1 & 1 \\
0 & 0
\end{array}\right], \quad b=\left[\begin{array}{ll}
1 & 0 \\
0 & 1
\end{array}\right]
$$

it is easy to see that $a \leq_{\diamond} b$ and however $a \not^{\#} b$.

Defining $a^{\pi, r}=1-a a^{\dagger}$ and $a^{\pi, l}=1-a^{\dagger} a$ we obtain:

Lemma 7 Let $R$ be a ring with unity and $a, b \in R$. Then the following conditions are equivalent:

(a) $a \leq_{\diamond} b$

(b) $b^{\pi, r} \leq_{\diamond} a^{\pi, r}, b^{\pi, l} \leq_{\diamond} a^{\pi, l}$ and $\left(1-a^{\pi, r}\right)\left(1-b a^{\dagger}\right)=0$.

(c) $b^{\pi, r} \leq^{-} a^{\pi, r}, b^{\pi, l} \leq^{-} a^{\pi, l}$ and $\left(1-a^{\pi, r}\right)\left(1-b a^{\dagger}\right)=0$.

Proof. We first observe that $\left(1-x x^{\dagger}\right)^{\dagger}=1-x x^{\dagger}$ and $\left(1-x^{\dagger} x\right)^{\dagger}=1-x^{\dagger} x$ for $x \in\{a, b\}$. Now, we apply Theorem 2. The following equivalences are valid:

$$
\begin{gathered}
a a^{\dagger} b b^{\dagger}=a a^{\dagger}=b b^{\dagger} a a^{\dagger} \Longleftrightarrow \\
\Longleftrightarrow 1-b b^{\dagger}=\left(1-a a^{\dagger}\right)\left(1-b b^{\dagger}\right)=\left(1-b b^{\dagger}\right)\left(1-a a^{\dagger}\right) \Longleftrightarrow 1-b b^{\dagger} \leq^{-} 1-a a^{\dagger} .
\end{gathered}
$$

Similarly, it can be shown that $b^{\dagger} b a^{\dagger} a=a^{\dagger} a$ is equivalent to $b^{\pi, l} \leq_{\diamond} a^{\pi, l}$ and $a^{\dagger} b a^{\dagger}=a^{\dagger}$ is equivalent to $\left(1-a^{\pi, r}\right)\left(1-b a^{\dagger}\right)=0$. Hence, (a) $\Longleftrightarrow(\mathrm{b})$. The equivalence between (b) and (c) follows directly from Theorem 2 .

\section{Successors and predecessors under the di- amond partial order}

Let us start this section with a result valid for the minus partial order. 
Lemma 8 Let $x, y \in R$. Then the following conditions are equivalent:

(a) $x \leq^{-} y$.

(b) There exists $x^{=} \in x\{1,2\}$ such that $y-x \in{ }^{\circ}\left(x^{=}\right) \cap\left(x^{=}\right)^{\circ}$.

Proof. If $x \leq^{-} y$ then $x^{-} x=x^{-} y$ and $x x^{-}=y x^{-}$for some $x^{-} \in x\{1\}$. Taking $x^{=}=x^{-} x x^{-}$we have that $x^{=} \in x\{1,2\}$ with $x^{=} x=x^{=} y$ and $x x^{=}=$ $y x^{=}$. So, $(y-x) x^{=}=0$ and $x^{=}(y-x)=0$ and this last two equalities are equivalent to $y-x \in{ }^{\circ}\left(x^{=}\right) \cap\left(x^{=}\right)^{\circ}$.

The converse is trivial.

Given $a \in R$, in the following we find all the elements $b \in R$ such that $a \leq_{\diamond} b$. Such elements $b$ are called the successors of $a$.

Theorem 5 Let $a \in R$. Then the following conditions are equivalent:

(a) There exists $b \in R$ such that $a \leq_{\diamond} b$.

(b) There exists $h \in{ }^{\circ}\left(\left(a^{\dagger}\right)^{=}\right) \cap\left(\left(a^{\dagger}\right)^{=}\right)^{\circ}$ such that $b=\left(a^{\dagger}+h\right)^{\dagger}$.

Proof. If $a \leq_{\diamond} b$ then $a^{\dagger} \leq^{-} b^{\dagger}$. Taking $x=a^{\dagger}, y=b^{\dagger}$ in Lemma 8 we have that $(\mathrm{a}) \Longrightarrow(\mathrm{b})$. The converse is evident.

Given $b \in R$, all the elements $a \in R$ such that $a \leq_{\diamond} b$ are called the predecessors of $b$. A partial solution of the problem of finding all the predecessors of a fixed element is given in the following result.

Theorem 6 Let $b, h \in R$. If $(b-h)^{\dagger}=b^{\dagger}-h^{\dagger}$ and $h \leq^{*} b$ then $a=b-h$ satisfies $a \leq_{\diamond} b$.

Proof. Since $h \leq^{*} b$, we have $b h^{\dagger} h=h=h h^{\dagger} b$. Hence, we get $a a^{\dagger}=a b^{\dagger}$ and $a^{\dagger} a=b^{\dagger} a$ since

$$
\begin{aligned}
& a a^{\dagger}=(b-h)(b-h)^{\dagger}=(b-h) b^{\dagger}-b h^{\dagger}+h h^{\dagger}=a b^{\dagger}, \\
& a^{\dagger} a=(b-h)^{\dagger}(b-h)=b^{\dagger}(b-h)-h^{\dagger} b+h^{\dagger} h=b^{\dagger} a .
\end{aligned}
$$

Now, Theorem 2 ends the proof.

Other method to find predecessors of a given element $b \in R$ has been stated in Theorem 3 where the idempotents in the ring have to be previously found.

Theorem 6 allows us to state a similar result to that in Lemma 4 for the diamond partial order as follows. 
Theorem 7 Let $a, b \in R$ such that $(b-a)^{\dagger}=b^{\dagger}-a^{\dagger}$. Then $b-a \leq^{*} b$ iff $a \leq_{\diamond} b$.

Proof. By definition of star partial order and the assumption we have that $b-a \leq^{*} b$ holds iff $\left(b^{\dagger}-a^{\dagger}\right)(b-a)=\left(b^{\dagger}-a^{\dagger}\right) b$ and $(b-a)\left(b^{\dagger}-a^{\dagger}\right)=b\left(b^{\dagger}-a^{\dagger}\right)$. Some computations leads to $a^{\dagger} a=b^{\dagger} a$ and $a a^{\dagger}=a b^{\dagger}$. Theorem 2 yields $a \leq_{\diamond} b$. The converse can be shown in a similar way.

Corollary 2 If $(b-a)^{\dagger}=b^{\dagger}-a^{\dagger}$ for all element $a, b \in R$ then the star and diamond partial orders coincide, that is $a \leq^{*} b$ iff $a \leq_{\diamond} b$.

Theorem 8 Let $b \in R$. The following statements are valid:

(a) If $b^{\dagger}$ is idempotent then $a \leq_{\diamond} b$ iff $\left(a^{\dagger}\right)^{2}=a^{\dagger}=a^{\dagger} b^{\dagger}=b^{\dagger} a^{\dagger}$.

(b) If $b$ is hermitian idempotent then $a \leq_{\diamond} b$ iff $\left(a^{\dagger}\right)^{2}=a^{\dagger}=a^{\dagger} b=b a^{\dagger}$.

Proof. (a) It follows directly from Lemma 3 taking $x=a^{\dagger}, y=b^{\dagger}$ and Theorem 2.

(b) It is a particular case of (a).

Now we characterize the intervals

$$
\left[0, a^{\pi, r}\right]=\left\{b \in R: 0 \leq_{\diamond} b \leq_{\diamond} a^{\pi, r}\right\} \text { and }\left[0, a^{\pi, l}\right]=\left\{b \in R: 0 \leq_{\diamond} b \leq_{\diamond} a^{\pi, l}\right\}
$$

for a fixed element $a \in R$.

Proposition 3 Let $a \in R$. Then

$$
\left[0, a^{\pi, r}\right]=\left\{b \in R: b^{\dagger} \text { is idempotent and } b^{\dagger} \in{ }^{0} a \cap\left(a^{\dagger}\right)^{0}\right\} .
$$

and

$$
\left[0, a^{\pi, l}\right]=\left\{b \in R: b^{\dagger} \text { is idempotent and } b^{\dagger} \in{ }^{0}\left(a^{\dagger}\right) \cap a^{0}\right\} .
$$

Proof. Since $a^{\pi, r}$ is idempotent and hermitian, we apply Theorem 8 and then $b \leq_{\diamond} a^{\pi, r}$ iff $\left(b^{\dagger}\right)^{2}=b^{\dagger}=b^{\dagger} a^{\pi, r}=a^{\pi, r} b^{\dagger}$. Some algebraic manipulations show that these last equalities are equivalent to $b^{\dagger}$ is idempotent and $a^{\dagger} b^{\dagger}=b^{\dagger} a=0$. Similarly for the idempotent $a^{\pi, l}$. 
Lemma 9 If $u \in R$ is a unit then $u$ is maximal under the diamond partial order.

Proof. Given a unit $u$ and an arbitrary $a \in R$, if $u \leq_{\diamond} a$ then $u^{-1} \leq^{-} a^{\dagger}$. On account of [5, Proposition 3 (i)], $a^{\dagger}=u^{-1}$ from which $a=u$ is maximal.

Theorem 9 Let $a \in R^{\#}$. Then a is a unit iff a is maximal of $R^{\#}$ under the diamond partial order.

Proof. $(\Longrightarrow)$ It follows directly from Lemma 9 .

$(\Longleftarrow)$ Take $u=a+1-a a^{\dagger}$ with $a \in R^{\#}$. Then $u$ is a unit with $u^{-1}=a^{\#}+1-a a^{\dagger}$. Therefore, $a R \subseteq u R=R$ and $R a \subseteq R u=R$. Also, $a a^{*} a=a u^{*} a$. These mean $a \leq_{\diamond} u$. Since $a$ is maximal then $a=u$ is a unit and the result follows.

\section{References}

[1] J.K. Baksalary, O.M. Baksalary, X. Liu, Further properties of the star, left-star, right-star, and minus partial orderings, Linear Algebra and its Applications, 375, 83-94, 2003.

[2] J.K. Baksalary, O.M. Baksalary, X. Liu, G. Trenkler, Further results on generalized and hypergeneralized projectors, Linear Algebra and its Applications, 429, 1038-1050, 2008.

[3] J.K. Baksalary, J. Hauke, A further algebraic version of Cochran's theorem and matrix partial orderings, Linear Algebra and its Applications, 127, 157-169, 1990.

[4] B. Blackwood, S.K. Jain, K.M. Prasad, A.K. Srivastava, Shorted Operators Relative to a Partial Order in a Regular Ring, arXiv:1010.0051v1.

[5] R.E. Hartwig, How to partially order regular elements, Mathematica Japonica, 25, 1, 1-13, 1980.

[6] J. Hauke, A. Markiewicz, T. Szulc, Inter-and extrapolatory properties of matrix partial orderings, Linear Algebra and its Applications, 332-334, 437-445, 2001. 
[7] S. Malik, Matrix partial orders and reversal law, Indian Journal of Pure and Applied Mathematics, 38, 6, 569-577, 2007.

[8] S. K. Mitra, P. Bhimasankaram, S.B. Malik, Matrix partial orders, shorted operators and applications. World Scientific Publishing Company, 2010.

[9] P. Patrício, C. Mendes Araujo. Moore-Penrose invertibility in involutory rings: the case $a a^{\dagger}=b b^{\dagger}$, Linear and Multilinear Algebra, 58, 4, 445-452, 2010 .

[10] C.R. Rao, S.K. Mitra, Generalized inverse of matrices and its applications. John Wiley \& Sons, Inc., New York-London-Sydney, 1971.

[11] D. Mošić, D. S. Djordjević, Some results on the reverse order law in rings with involution, Aequationes Mathematicae, 83, 3, 271-282, 2012.

[12] D. Mošić, D. S. Djordjević, Further results on the reverse order law for the Moore-Penrose inverse in rings with involution, Applied Mathematics and Computation, 218, 4, 1478-1483, 2011.

[13] M. Tošić, D.S. Cvetković-Ilić, Invertibility of a linear combination of two matrices and partial orderings, Applied Mathematics \& Computations, 218, 4651-4657, 2012. 